\title{
Possible Dynamic States in Inductively Coupled Intrinsic Josephson Junctions of Layered High- $T_{c}$ Superconductors
}

\author{
Shizeng $\mathrm{Lin}^{1,2}$ and $\mathrm{Xiao} \mathrm{Hu}^{1,2,3}$ \\ ${ }^{1}$ WPI Center for Materials Nanoarchitectonics, National Institute for Materials Science, Tsukuba 305-0047, Japan \\ ${ }^{2}$ Graduate School of Pure and Applied Sciences, University of Tsukuba, Tsukuba 305-8571, Japan \\ ${ }^{3}$ Japan Science and Technology Agency, 4-1-8 Honcho, Kawaguchi, Saitama 332-0012, Japan
}

(Dated: November 21, 2018)

\begin{abstract}
Based on computer simulations and theoretical analysis, a new dynamic state is found in inductively coupled intrinsic Josephson junctions in the absence of an external magnetic field. In this state, the plasma oscillation is uniform along the $c$ axis with the fundamental frequency satisfying the ac Josephson relation. There are $(2 m+1) \pi$ phase kinks around the junction center, with $m$ being an integer, periodic and thus non-uniform in the $c$ direction. In the IV characteristics, the state manifests itself as current steps occurring at all cavity modes. Inside the current steps, the plasma oscillation becomes strong, which generates several harmonics in frequency spectra at a given voltage. The recent experiments on terahertz radiations from the mesa of a BSCCO single crystal can be explained in terms of this state.
\end{abstract}

PACS numbers: 74.50.+r, 74.25.Gz, 85.25.Cp

Soon after the discovery of high- $T_{c}$ superconductivity in cuprates, it was demonstrated that a single crystal of layered superconductors, such as BSCCO, behaves as a stack of intrinsic Josephson junctions (IJJs) [1]. The IJJs have been investigated extensively since then, and various dynamical properties were explored. One focus in this field is to exploit the dynamic states of IJJs to generate electromagnetic waves in the terahertz $(\mathrm{THz})$ regime 2, 3, 4]. The high- $T_{c}$ cuprate superconductors are unique for this purpose since the IJJs are homogeneous at atomic scale and the energy gap is large, $60 \mathrm{meV}$, which make it possible to tune the frequency in a wide range by adjusting the bias voltage. To obtain coherent radiations, however, all the junctions should be operated in-phase, which turns out to be not easy.

The dynamics of IJJs are appropriately described by the coupled sine-Gorden equations [5]

$$
\partial_{x}^{2} P_{l}=\left(1-\zeta \Delta^{(2)}\right)\left(\sin P_{l}+\beta \partial_{t} P_{l}+\partial_{t}^{2} P_{l}-J_{\text {ext }}\right),
$$

where $P_{l}$ is the gauge-invariant phase difference at the $l$ th junction, $\beta \equiv 4 \pi \sigma_{c} \lambda_{c} / c \sqrt{\varepsilon_{c}}$ the normalized $c$-axis conductivity, $\zeta \equiv \lambda_{a b}^{2} / s D$ the inductive coupling and $\Delta^{(2)} P_{l} \equiv P_{l+1}+P_{l-1}-2 P_{l}$ the difference operator. $\varepsilon_{c}$ is the dielectric constant and $\sigma_{c}$ is the conductance along the $c$ axis. $\lambda_{c}$ and $\lambda_{a b}$ are the penetration depths. $s(D)$ is the thickness of the superconducting (insulating) layer. Length is normalized by $\lambda_{c}$, time is normalized by the Josephson plasma frequency $1 / 2 \pi f_{J}$ with $f_{J} \equiv c / 2 \pi \lambda_{c} \sqrt{\varepsilon_{c}}$, and voltage is in unit of $\Phi_{0} f_{J} / c$. Here $c$ is the light velocity in vacuum.

Without an external in-plane magnetic field, Eq. (1D) have the plasma solution, moving soliton and anti-soliton states, and McCumber state [6]. The plasma solution corresponds to the collective small phase oscillation in Eq. (1). It resonates in the cavity formed by IJJs when the cavity resonance condition meets, i.e. $q \lambda_{w} / 2=L$ with q being an integer, $\lambda_{w}$ plasma wavelength and $L$ the length of the IJJs. It corresponds to the voltage per layer $V=q \pi c_{j} / L$ with $c_{j}$ the plasma velocity $(1 \leq j \leq N, N$ the total number of junctions) [7] assuming that the ac Josephson relation is satisfied.

In the soliton state, a soliton (anti-soliton) moving with velocity $v$ is reflected as an anti-soliton (soliton) by the edges. The periodic motion and reflection of soliton and anti-soliton contributes to a dc voltage $V=2 \pi v / L$. When the velocity of soliton approaches $c_{j}$, it gives birth to the zero-field step (ZFS) at $V=2 \pi c_{j} / L[6,6,8$, 9], which corresponds to the 2 nd cavity mode. If $n$ solitons engage in this process, they produce the $n$th ZFS. The interplay between solitons and plasma leads to many peculiar behaviors [10], such as Cherenkov radiation [11].

In the McCumber state, the phase is uniform through the $x$ direction. Therefore the junctions are decoupled and it gives linear branches in the IV characteristics.

In the present study, we find that in the absence of an external magnetic field, a new dynamic state can be achieved in the inductively coupled sine-Gordon systems, which is characterized as follows: in the IV characteristics it generates current steps at all cavity modes; while the plasma oscillation is uniform along the $c$ axis, in the total phase there are static kinks of $(2 m+1) \pi$ along the $x$ axis, which are periodic but non-uniform in the $c$ direction. It is shown that the recent experiments [3, 4] on terahertz emission can be understood in terms of this new state.

The results shown below are for $\beta=0.02, \zeta=4 / 9 \times$ $10^{6}$, typically for BSCCO, and $L=80 \mu \mathrm{m}$; confirmations of the main results have been made with other parameters. As displayed in the inset of Fig. 1, the current $J_{\text {ext }}$ is fed in along the $c$ axis. Since we are searching for states periodic in the $c$ axis, we adopt the periodic boundary condition along this direction which makes the maximum plasma velocity $c_{0}=1$ possible. The equa- 

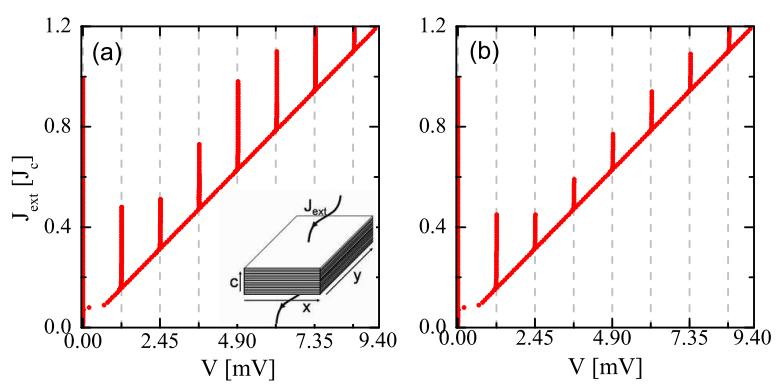

FIG. 1: (color). Simulated IV characteristics under the boundary conditions (a) without and (b) with radiation with $C=0.000177$ and $R=707.1$. The vertical dashed lines correspond to the voltage at cavity modes $V=n \Phi_{0} /\left(2 \sqrt{\varepsilon_{c}} L\right)$. Inset: geometry of the mesa of BSCCO single crystal.

tions of motion Eq. (11) are integrated by the staggered leapfrog algorithm. The time step in all simulations is set to $\Delta t=0.0018$ and the mesh size is set to $\Delta x=0.002$. The accuracy is checked with smaller $\Delta x$ and $\Delta t$. We consider first the system under the boundary condition $\partial_{x} P_{l}=0$, which turns out to be a good unperturbed state for discussion on radiation as revealed later.

The important feature of the IV characteristics in Fig. 1(a) is the existence of current steps. In sharp contrast with the ZFS in single junctions [8], the present current steps in IJJs occur at both even and odd cavity modes; the dc voltage and the frequency of plasma oscillation satisfy the ac Josephson relation.

As revealed by the present numerical simulations, there are four parts in the phase variables: (1) a term evolving linearly with time according to the ac Josephson relation $\omega t,(2)$ a term of $(2 m+1) \pi$ phase kinks and anti-kinks arranged periodically in the $c$ direction, (3) a plasma term uniform along the c axis $\widetilde{P}(x, t),(4)$ a constant term $\varphi$. Figure 2 exhibits several typical configurations for the 2nd term. For ease of discussions, we restrict ourselves
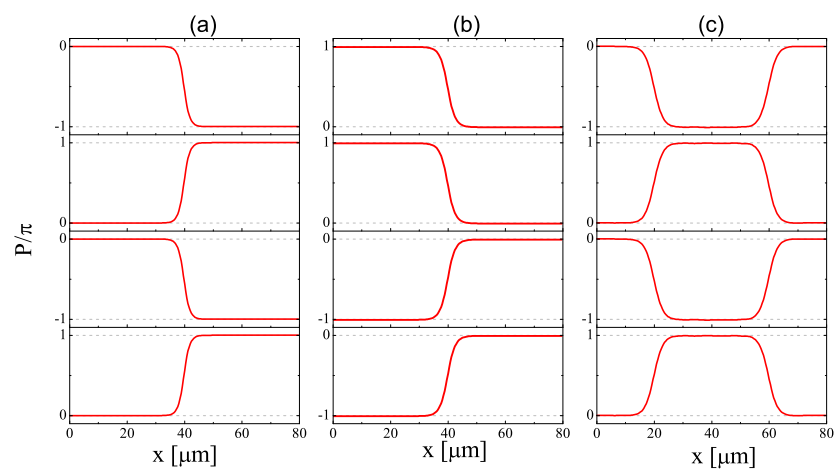

FIG. 2: (color). Typical configurations of the static term in the phase difference (the 2nd term in Eq. (2)): (a) period of 2 layers and (b) period of 4 layers, corresponding to the 1st current step, and (c) corresponding to the 2nd current step. to the 1st cavity mode in the following discussions, which can be extended to higher modes straightforwardly. The total phase therefore can be expressed by

$$
P_{l}(x, t)=\omega t+f_{l} P_{l}^{s}(x)+\widetilde{P}(x, t)+\varphi,
$$

where $P_{l}^{s}(x)$ is a soliton from 0 to $2 \pi$ with the center at $x=L / 2$ (see below), $\widetilde{P}(x, t)=A \cos (\pi x / L) \sin \omega t+$ high harmonics.

Substituting Eq. (2) into Eq. (11) and using the expansion of sine of sine by the Bessel function, we arrive at the following relation for the static parts of phases

$$
\begin{aligned}
& f_{l} \partial_{x}^{2} P_{l}^{s}=\omega \beta-J_{\text {ext }} \\
& -\left(1-\zeta \Delta^{(2)}\right) J_{1}(A \cos (\pi x / L)) \sin \left(f_{l} P_{l}^{s}+\varphi\right),
\end{aligned}
$$

where $J_{1}$ is the Bessel function of the first kind and for simplicity only the fundamental mode is taken.

Off resonance the system behaves in an ohmic way as seen in Fig. 1, where $J_{\text {ext }} \approx \beta \omega$ and $\varphi \approx 0$. The solutions of period of 2 layers (period-2), where $f_{l}=(-1)^{l} f$ (Fig. 22(a)), and of 4 layers (period-4), where $f_{l}=(-1)^{[l / 2]} f$ (Fig. 2(b)), diagonalize the difference operator $\Delta^{(2)}$. In both cases, $P_{l}^{s}$ is independent of $l$, thus $P_{l}^{s}=P^{s 0}$. We then arrive at the equation

$$
f \partial_{x}^{2} P^{s 0}=-(1+q \zeta) J_{1}(A \cos (\pi x / L)) \sin \left(f P^{s 0}\right),
$$

where $q=4$ for period-2 and $q=2$ for period- 4 .

Since the l.h.s. of Eq. (4) and $J_{1}(A \cos (x \pi / L))$ are antisymmetric with respect to $x=L / 2, \sin \left(f P^{s 0}\right)$ must be symmetric. This imposes $f=(2 m+1) / 2$, with an integer $m$.

When the voltage is tuned to one of the cavity modes, the plasma oscillation can acquire large amplitude in the form of standing waves. By taking a non-zero constant $\varphi$, the system can bypass increasing $J_{\text {ext }}$ through the Josephson current

$$
J_{\text {ext }}=\beta \omega-\frac{\sin \varphi}{L} \int_{0}^{L} d x J_{1}(A \cos (\pi x / L)) \cos \left(f P^{s 0}\right) .
$$

This gives rise to the current steps in the IV characteristics in Fig. 1 Extra amount of energy besides the ohmic dissipation is fed into the plasma oscillation.

In Figs. 3 (a)-(c), we display distributions of the dc part of Josephson current $\left\langle\sin P_{l}\right\rangle_{t}$ in two adjacent junctions. The symbols are given by $J_{1}(A \cos (\pi x / L)) \sin \left( \pm P^{s 0}(x) / 2+\varphi\right)$ where $P^{s 0}(x)=$ $4 \arctan \left[\exp \left((x-L / 2) / \lambda_{J}^{\prime}\right)\right]$ (see Eq. (4) $)$. The dependence of net Josephson current $J_{\text {net }} \equiv\left\langle\sin P_{l}\right\rangle_{x, t}$ on $J_{\text {ext }}$ at the 1st current step is shown in Fig. 3(d).

The remaining terms in the r.h.s. of Eq. (3), which are proportional to the inductive coupling $\zeta$, should correspond to a (unquantized) soliton as required by the l.h.s of the equation. This can be seen clearly in Fig. 3(e). The system arranges itself in a way that the parameter $\zeta$ is blocked from involving directly in the net Josephson current, which should be in order of $J_{c}$. 

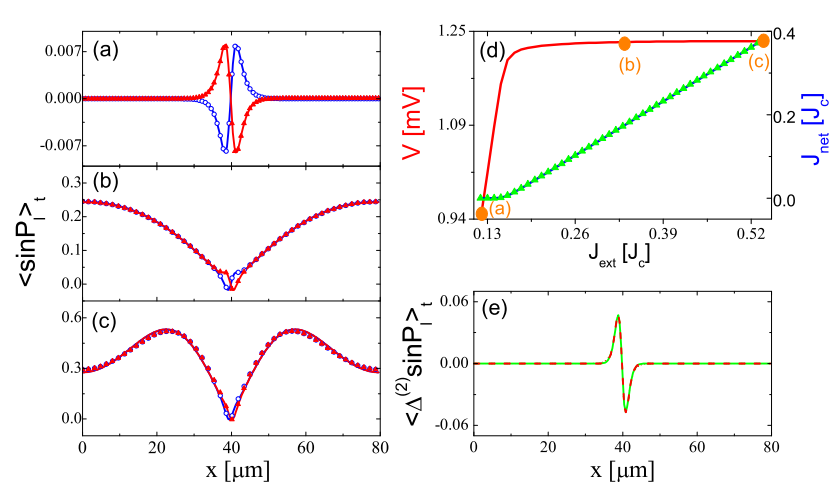

FIG. 3: (color). Comparison between the theory and simulations on the dc part of Josephson current $\left\langle\sin P_{l}\right\rangle_{t}$ in two neighboring layers for the configuration in Fig. 2(b): (a) off resonance at $J_{\text {ext }}=0.12$, (b) at the middle of the 1st step with $J_{\text {ext }}=0.35$, (c) at the top of the 1st step with $J_{\text {ext }}=0.53$. The solid curves are results by numerical simulations and the symbols are derived by the analytic expressions. (d) Current dependence of voltage (red) and $J_{\text {net }}$ (blue) at the 1st current step. Green symbols are for the theoretical estimate $J_{\text {ext }}-\omega \beta$. (e) $\left\langle\Delta^{(2)} \sin P_{l}\right\rangle_{t}$ at $J_{\text {ext }}=0.35$ (dashed red line) and at $J_{\text {ext }}=0.53$ (dashed green line).

Despite that the total phase is non-uniform along the $c$ axis, the electric field $E_{l}^{z}=\partial_{t} P_{l}$ as well as the magnetic field $B_{l}^{y}=\left(1-\zeta \Delta^{(2)}\right)^{-1} \partial_{x} P_{l}=\partial_{x} \widetilde{P}+O(1 / \zeta)$ with $P_{l}(x, t)$ given by Eq. (2) are uniform along the $c$ axis. This makes a coherent superradiation possible as observed in the recent experiments [3, 4] . Distributions
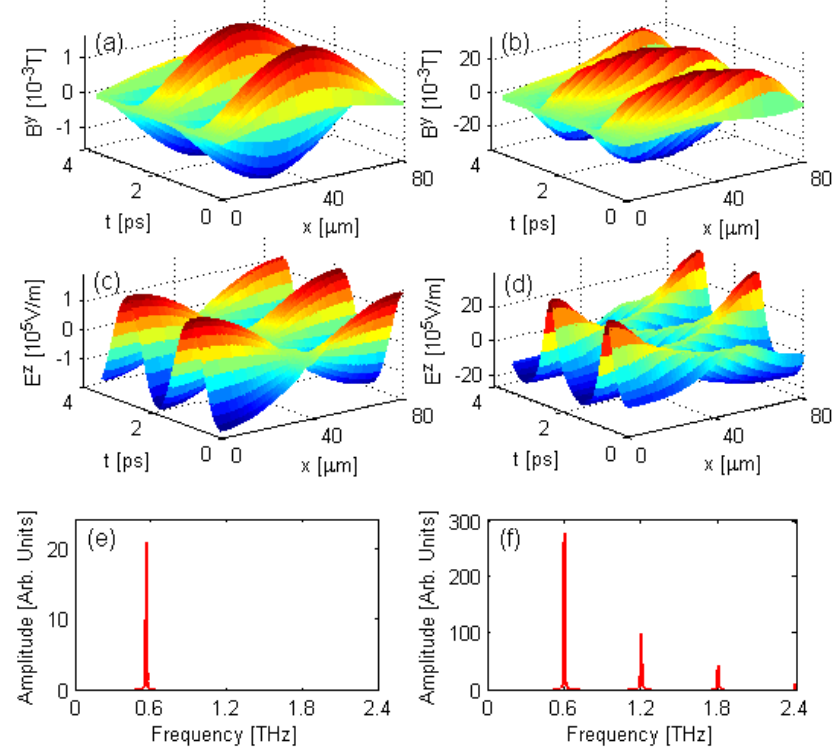

FIG. 4: (color). Time evolutions of the magnetic field and electric field and the frequency spectrum. The dc part of the electric field has been subtracted. Left/right: at the bottom/top of the 1st current step. of electric and magnetic fields are displayed in Fig. 4, In contrast with the state at bottom of the current step (left of Fig. (4), the amplitude of plasma term increases and high harmonics become visible inside the current steps, and thus the standing waves of electromagnetic field are not simply trigonometric anymore (right of Fig. 4). The wave crest of magnetic field becomes smaller when it approaches the edges and it changes sign when it is reflected. It resembles the motion of solitons in ZFS except that the magnetic flux is not quantized since it is associated with the plasma oscillations.

From the foregoing minimum theoretical analysis, we have already captured the main features of the system revealed by simulations. The following discussions are necessary to make the picture more comprehensive: (I) There are infinite number of configurations of the static phase even in the same current step. Besides those in Figs. 2(a) and (b), periodic configurations of $(2 m+1) \pi$ kinks, and their composites are observed in simulations. Configurations of phase kinks not periodic in the $c$ axis may be possible. Superpositions of soliton(s) and antisoliton(s) along the junction direction is also permitted as for the single sine-Gordon system [12]. The height of current steps depends on the specific configuration as can be read from Eq. (5). (II) The phase configurations for higher cavity modes can be constructed from the first cavity mode, see Fig. 2(d). (III) In addition to the plasma oscillation, there exists a small oscillation of the center of soliton $P_{l}^{s}(x)$ as well, which produces a spike-like oscillation with characteristic length $\lambda_{J}^{\prime}$ in electromagnetic fields. (IV) As revealed in the above discussions, the strong inductive coupling is essential for the new dynamic states, and therefore it is impossible to build such current steps in a single Josephson junction. In fact, the current steps completely vanish when $\zeta$ is small in simulations. (V)While BSCCO may be the best choice, the new state can be realized in other layered superconductors, including s-wave ones with not so high $T_{c}$. The crystal is to be thick to reduce the surface effect.

Let us investigate the possible radiation of energy from the IJJs. The boundary condition should be formulated explicitly for this purpose. Theoretical efforts have been attempted to model the interaction between IJJs and the outside space [13, 14, 15, 16]. We model the external load by an effective RC circuit [14], and hence the boundary condition becomes $\partial_{t} \widetilde{P} / \partial_{x} \widetilde{P}=\widetilde{E}^{z} / \widetilde{B}^{y}=$ $Z=R-i /(C \omega)=|Z| \exp (i \theta)$. The sharp radiation peaks upon voltage sweeping observed in recent experiments [3, 4] indicate that there is a significant mismatch in impedance between IJJs and the outside space, and that electromagnetic waves are reflected mostly at the edges of IJJs. The small ratio between the mesa thickness in the experiments and $\lambda_{c}$ may be a source of this mismatch [16], which implies $|Z| \gg 1$. We have confirmed that the new states remain stable for large values of $|Z|$. 


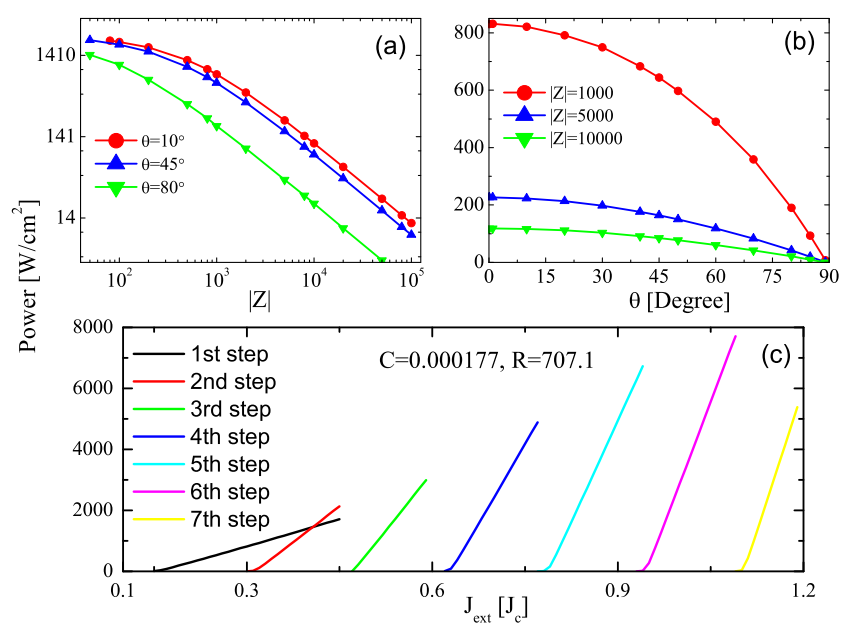

FIG. 5: (color). Dependence of radiation power evaluated by the Poynting vector at the edges of IJJs (a) on $|Z|$ and (b) on $\theta$ inside the 1 st current step at $J_{\text {ext }}=0.27$. (c) Radiation power at all current steps.

The IV characteristics under the boundary condition permitting radiation are shown in Fig. 1(b). The heights of the current steps are reduced when radiations are present, since when the radiation is strong it is hard to form stable standing waves.

As shown in Fig. [5(c), the radiation power increases linearly with the external current at the current steps. This behavior can be explained by the above theoretical description including additionally the radiation as a small perturbation. The power at the top of the 1st current step is of one order of magnitude stronger than that at the bottom, and the maximum power with the present boundary condition is about $8000 \mathrm{~W} / \mathrm{cm}^{2}$, which is quite promising for applications. The dependence of the radiation power on $|Z|$ and $\theta$ is detailed in Figs. 5(a) and (b), which can be described as $\cos \theta /|Z|$.

The above simulation results seem to be able to provide a consistent picture for the two recent experiments [3, 4] on the terahertz radiation in zero external magnetic field. Coherent radiations were detected in the resistive curve in Ref. [3] with the frequency corresponding to the fundamental mode of the 1st current step. In Ref. [4], radiations were detected in the anomalous region of IV curve, and the detected power is much stronger than that in Ref. 3]. Comparing these features with our simulated results, it is natural to assign the radiations in Ref. [4] to states inside the 1st current step, while those in Ref. [3] to states at the bottom of the current step. The shape of the anomalous IV curve where radiations are observed in Ref. [4] is also indicative that the system falls into the current step. High cavity modes could not be observed in experiments due to the heating effect, which is not taken into account in the present model. The temperature dependence of the conductivity $\beta$ is necessary in order to simulate the overall shape of IV characteristics in the experiments especially at high currents.

In contrast, the plasma solution without static kinks (crossover to the McCumber state without radiation) cannot show the sharp peak in radiation energy when voltage is tuned, and there is no current step because the plasma do not couple to the cavity modes. On the other hand, the radiation from the soliton state does not satisfy the ac Josephson relation, and thus it cannot explain the recent experiments.

In conclusion, based on computer simulations and theoretical analysis we reveal the existence of a new dynamic state in inductively coupled intrinsic Josephson junctions. In this state, the plasma oscillation is uniform along the $c$ axis with the fundamental frequency satisfying the ac Josephson relation, while static phase kinks of $(2 m+1) \pi$ are stacked periodically. The states manifest themselves in the IV characteristics as current steps at all cavity modes. This state supports quite strong radiations. The recent experiments on $\mathrm{THz}$ radiations from BSCCO single crystals can be interpreted in terms of this state in a consistent way .

The authors thank U. Welp, K. Kadowaki, M. Tachiki, L. Bulaevskii, A. Koshelev and N. Pederson for discussions. Calculations were performed on SR11000 (HITACHI) in NIMS. This work was supported by WPI Initiative on Materials Nanoarchitronics, MEXT, Japan, CREST-JST, Japan and ITSNEM of CAS.

[1] R. Kleiner et al., Phys. Rev. Lett. 68, 2394 (1992).

[2] T. Koyama and M. Tachiki, Solid State Commun. 96, 367 (1995); M. Tachiki et al., Phys. Rev. B 71, 134515 (2005); I. E. Batov et al., Appl. Phys. Lett. 88, 262504 (2006); K. Kadowaki et al., Physica C 437, 111 (2006); M.-H. Bae, et al., Phys. Rev. Lett. 98, 027002 (2007); L. N. Bulaevskii and A. E. Koshelev, Phys. Rev. Lett. 99, 057002 (2007); S. Z. Lin et al., Phys. Rev. B 77, 014507 (2008).

[3] L. Ozyuzer et al., Science 318, 1291 (2007).

[4] K. Kadowaki et al., preprint.

[5] S. Sakai et al., J. Appl. Phys. 73, 2411 (1993).

[6] R. Kleiner et al., Phys. Rev. B 62, 4086 (2000).

[7] S. Sakai et al., Phys. Rev. B 50, 12905 (1994).

[8] T. A. Fulton and R. C. Dynes, Solid State Commun. 12, 57 (1973).

[9] V. M. Krasnov and D. Winkler, Phys. Rev. B 60, 13179 (1999).

[10] A. V. Ustinov, Physica D 123, 315 (1998).

[11] G. Hechtfischer et al., Phys. Rev. Lett. 79, 1365 (1997).

[12] D. W. McLaughlin and A. C. Scott, Phys. Rev. A 18, 1652 (1978).

[13] N. Grønbech-Jensen et al., Phys. Lett. A 142, 427 (1989).

[14] C. Soriano et al., Supercond. Sci. Technol. 9, 578 (1996).

[15] L. N. Bulaevskii and A. E. Koshelev, Phys. Rev. Lett. 97, 267001 (2006).

[16] A. E. Koshelev and L. N. Bulaevskii, Phys. Rev. B 77, 
014530 (2008). 